International Journal of Pure and Applied Mathematics

Volume 104 No. 3 2015, 461-470

ISSN: 1311-8080 (printed version); ISSN: 1314-3395 (on-line version)

url: http://www.ijpam.eu

doi: http://dx.doi.org/10.12732/ijpam.v104i3.13

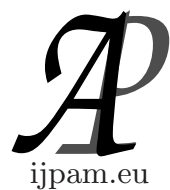

\title{
QUASI-COMPLETION OF FILTER SPACES
}

\author{
Nandita Rath \\ School of Mathematics and Statistics \\ University of Western Australia \\ Crawley, W.A. 6009, AUSTRALIA
}

\begin{abstract}
The category FIL of filter spaces being isomorphic to the category of grill-determined nearness spaces has become significant in the later part of the twentieth century. During that period, a substantial completion theory has been developed using the equivalence classes of filters in a filter space. However, that completion was quite general in nature, and did not allow the finest such completion. As a result, a completion functor could not be defined on FIL. In this paper, this issue is partially addressed by constructing a completion that is finer than the existing completions. Also, a completion functor is defined on a subcategory of FIL comprising all filter spaces as objects.
\end{abstract}

AMS Subject Classification: 54D35, 54A20, 54C20

Key Words: filter space, Cauchy map, convergence structure, $s$-map, stable completion, completion in standard form

\section{Introduction}

In 1990, Bently et al. [1] formalised the concept of filter spaces for being isomorphic to Katetov's [2] filter merotopic spaces. Since then these spaces have been studied by several topologists (see [3], [4], [5], [6], [7]) in the context of their applications to category theory and algebra . Kent and Rath [3] defined

Received: August 16, 2015

(c) 2015 Academic Publications, Ltd. url: www.acadpubl.eu 
an equivalence relation on a filter space $(X, C)$, which led to the construction of its $T_{2}$ Wyler completion. However, soon they realised that unlike the completion of Cauchy spaces, there is no finest completion when there are infinite number of equivalence classes (see Proposition 2.4 [3]). Attempts have been made in this paper to construct a certain type of weaker completion, called quasi completion of a filter space which may yield a finest such completion in a subcategory of FIL which has all filter spaces as objects.

Also, the well-known completion theory for Cauchy spaces was extended to obtain a completion without the $T_{2}$-restriction on the space by the author [9]. An $s$-map was introduced to form a special class of morphisms which led to a completion functor on a subcategory of $C H Y$ (the category of Cauchy spaces with Cauchy maps) with respect to a new class of morphisms. In this paper, a modified form of $s$-maps is used to build a completion functor on a subcategory of FIL (the category of filter spaces with Cauchy maps) without the $T_{2}$ restriction.

Reed [12] introduced a special type of completion for $T_{2}$ Cauchy spaces, namely completion in standard form, which was very interesting in the sense that it led to a powerful result: any $T_{2}$ Wyler completion is equivalent to one in standard form. However, as pointed out via a counter example by the author in an earlier paper [9, Example 3.2], this is not the case for all Cauchy spaces in general, that is, it fails to preserve the equivalence of completions in standard form, since it is not a categorical equivalence in the sense of Preuss [8]. Since Cauchy spaces are special cases of filter spaces, Reed's completion will also fail to preserve the equivalence, for non- $T_{2}$ filter spaces in general. This motivates the introduction of quasi-stable completion.

\section{Preliminaries}

Let $X$ be a nonempty set and $\mathbf{F}(X)$ be the set of filters on $X$. If $\mathcal{F}$ and $\mathcal{G} \in F(X)$ and $F \cap G \neq \phi$ for all $F \in \mathcal{F}$ and $G \in \mathcal{G}$, then $\mathcal{F} \vee \mathcal{G}$ denotes the filter generated by $\{F \cap G: F \in \mathcal{F}$ and $G \in \mathcal{G}\}$. If there exist $F \in \mathcal{F}$ and $G \in \mathcal{G}$ such that $F \cap G=\phi$, then we say that $\mathcal{F} \vee \mathcal{G}$ fails to exist. For each $x \in X, \dot{x}$ denotes the ultrafilter generated by $\{x\}$. If $C \subset \mathbf{F}(X)$ satisfies the following conditions:

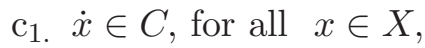

$\mathrm{c}_{2} . \mathcal{F} \in C$ and $\mathcal{G} \geq \mathcal{F}$ imply that $\mathcal{G} \in C$,

then the pair $(X, C)$ is called a filter space and $C$ is called a pre-Cauchy structure on $X$. If $C$ and $D$ are two pre-Cauchy structures on $X$, and $C \subseteq D$ 
then $C$ is finer than $D$, written $C \geq D$. Associated with each pre-Cauchy structure $C$ on a set $X$, there is a convergence structure $q_{c}$, defined as

$$
\mathcal{F} \stackrel{q_{c}}{\rightarrow} x \quad \text { if and only if } \quad \mathcal{F} \cap \dot{x} \in C .
$$

The two filters $\mathcal{F}$ and $\mathcal{G} \in \mathbf{F}(X)$ are said to be $C$-linked [3], if there exist a finite number of filters $\mathcal{H}_{1}, \mathcal{H}_{2}, \ldots, \mathcal{H}_{n} \in C$ such that $\mathcal{F} \vee \mathcal{H}_{1}, \mathcal{H}_{1} \vee \mathcal{H}_{2}, \ldots$, $\mathcal{H}_{n-1} \vee \mathcal{H}_{n}$ all exist. In particular, if $\mathcal{F}$ and $\mathcal{G} \in C$, we write $\mathcal{F} \sim_{c} \mathcal{G}$ iff $\mathcal{F}, \mathcal{G}$ are $C$-linked. A filter space is said to be a $c$-filter space (respectively, Cauchy space), if $\mathcal{F} \cap \dot{x} \in C$ whenever $\mathcal{F} \sim_{c} \dot{x}$ (respectively, $\mathcal{F} \cap \mathcal{G} \in C$ whenever $\mathcal{F} \sim_{c} \mathcal{G}$ ). Note that ' $\sim_{c}$ ' defines an equivalence relation on $C$. For $\mathcal{F} \in C$, let $[\mathcal{F}]_{c}$ denote the equivalence class containing $\mathcal{F}$. There is a pre-convergence structure [4] $p_{c}$ associated with $C$ in a natural way: $\mathcal{F} \stackrel{p_{c}}{\rightarrow} x$ iff $\mathcal{F} \sim_{c} \dot{x}$. Note that $p_{c} \leq q_{c}$ [3], since $\mathcal{F} \stackrel{q_{c}}{\rightarrow} x$ implies $\mathcal{F} \sim_{c} \dot{x}$, but $p_{c} \neq q_{c}$ in general, as illustrated in the following example.

Example 1. Let $X=R$, the set of real numbers and

$$
C=\{\dot{x} \mid x \in X\} \cup\{\mathcal{F} \mid \mathcal{F} \geq \mathcal{G}\} \cup\{\text { all free filters }\} .
$$

Clearly, $C$ is a pre-Cauchy structure on $X$. Consider the filter $\mathcal{H}=\{R \backslash F \mid$ $F$ is a finite subset of $R\}$. Since $\mathcal{F}$ is a free filter, it is in $C$. Observe that for the filter $\mathcal{G}=[\{[0,1 / n] \mid n \in N\}], \mathcal{H} \vee \mathcal{G}$ and $\mathcal{G} \vee \dot{0}$ exist, which imply that $\mathcal{H} \stackrel{p_{C}}{\rightarrow}$ 0. However $\mathcal{F} \cap \dot{0} \notin C$.

Lemma 1. For a filter space $(X, C), p_{c}=q_{c}$ if and only if it is a $c$-filter space.

A filter space $(X, C)$ is said to be quasi- $T_{1}$ (respectively, quasi- $\left.T_{2}\right)$ iff $\dot{x} \cap \dot{y} \in C \Rightarrow x=y$ (respectively, $\mathcal{F} \cap \dot{x}, \mathcal{F} \cap \dot{y} \in C \Rightarrow x=y$ ). Henceforth, the term "quasi" associated with any property for a filter space will be abbreviated to $q$-property, for example, quasi- $T_{1}$ will be referred to as $q-T_{1}$. The filter space is $q$-regular iff $c l_{q_{c}} \mathcal{F} \in C$ whenever $\mathcal{F} \in C$ and $q-T_{3}$ iff it is $q-T_{1}$ and $q$-regular.

Note that the properties such as $T_{1}, T_{2}, T_{3}$ and regularity of a filter space ( $X, C$ are stronger than the properties $q-T_{1}, q-T_{2}, q-T_{3}$ and $q$-regularity, respectively. It follows from Lemma 1 that these properties are equivalent only when $(X, C)$ is a $c$-filter space.

However, these properties shouldn't be undermined, since the quasi-properties of $(X, C)$ guarantee the corresponding properties of the convergence space $\left(X, q_{c}\right)$. For instance, $(X, C)$ is $q-T_{1}$ (respectively, $q-T_{2}, q-T_{3}$ and $q$-regular) implies that $\left(X, q_{c}\right)$ is $T_{1}$ (respectively, $T_{2}, T_{3}$ and regular). Moreover, if $\left(X, q_{c}\right)$ is regular, and every filter in $C q_{c}$-converges, then $(X, C)$ is regular. 
One of the remarkable differences between these properties and the weaker quasi-properties is that the $T_{1}$ and $T_{2}$ properties are equivalent [9] for a filter space, whereas this is not true in general for $q-T_{1}$ and $q-T_{2}$. The following example shows that there is a filter space which is $q-T_{1}$, but not necessarily $q-T_{2}$.

Example 2. Let $(X, C)$ be an infinite set and $a, b \in X$ such that $a \neq b$. Let

$$
C=\{\dot{x} \mid x \in X\} \cup\{\mathcal{G} \mid \mathcal{G} \geq \mathcal{H} \text { or } \mathcal{H} \cap \dot{a} \text { or } \mathcal{H} \cap \dot{b}\}
$$

where $\mathcal{H}$ is any filter on $X$. Clearly, $(X, C)$ is a filter space. For any $x, y$ in $X$, $\dot{x} \cap \dot{y} \in C \Rightarrow \dot{x} \cap \dot{y}$ is a fixed ultra-filter generated by a single element in $X$, which means $x=y$. So, $X$ is $q-T_{1}$. However, it is not $q-T_{2}$ since $\mathcal{H} \cap \dot{a}$ and $\mathcal{H} \cap \dot{b} \in C$, but $a \neq b$.

Note that $q-T_{1}$ and $q-T_{2}$ properties are equivalent for a $c$-filter space.

\section{Quasi-Completion}

Though the completion obtained by Kent and Rath [3] is the most general one, one of its drawback is that it does not have the finest such completion. As a result, a completion functor could not be defined on the category FIL of all filter spaces. In this section, a different completion is constructed, which yields such a functor on a subcategory of FIL. The $T_{2}$ Wyler completion of a filter space $(X, C)$ that was constructed by Kent and Rath [3] had the property that if $(X, C)$ was a $c$-filter space (Respectively, Cauchy space), then its completion was also $c$-filter space (respectively, Cauchy space). However, this is not the case for a quasi-completion.

For two filter spaces $(X, C)$ and $(Y, D)$, a mapping $f:(X, C) \longrightarrow(Y, D)$ is called a Cauchy map, if $\mathcal{F} \in C$ implies $f(\mathcal{F}) \in D$ for all $\mathcal{F} \in C$, and it is called a Cauchy embedding if $f:(X, C) \longrightarrow\left(f(X), D_{f(X)}\right)$ is bijective and both $f$ and $f^{-1}$ are Cauchy maps.

A filter space $(X, C)$ is said to be quasi-complete (respectively, complete) iff each $\mathcal{F} \in C \quad q_{c}$-converges (respectively, $p_{c}$-converges). In view of Example 1 , it follows that every quasi-complete filter space is complete, but not conversely. A quasi-completion of a filter space $(X, C)$ is a pair $((Y, D), \psi)$ consisting of a quasi-complete filter space $(Y, D)$ and a Cauchy embedding map $\psi:(X, C) \longrightarrow$ $(Y, D)$ satisfying $c l_{q_{D}} \psi(X)=Y$. A quasi-completion $((Y, D), \psi)$ is said to be a quasi- $\mathcal{P}$ completion, if $(Y, D)$ has the property $\mathcal{P}$ whenever $(X, C)$ has the same property. It is said to be $q$-proper, if images of any two equivalent filters in $C$ 
$q_{D}$-converge to the same point in $Y$.

Proposition 1. Any $q-T_{2}$ quasi-completion of a $q-T_{2}$ filter space is $q$ proper.

We construct a quasi-completion of a filter space $(X, C)$ as follows:

$X_{1}^{*}=X \cup\{[\mathcal{F}] \mid \mathcal{F} \in C\}, \mathcal{F} \nsim_{c} \dot{x}$ for any $\left.x \in X\right\}$,

$j: X \longrightarrow X_{1}^{*}$ is the inclusion map,

$C_{1}^{*}=j(C) \cup\left\{\mathcal{A} \in \mathbf{F}\left(X_{1}^{*}\right) \mid\right.$ there exists a filter $\mathcal{F} \in C$ such that $\mathcal{A} \geq$ $j(\mathcal{F}) \cap[\dot{\mathcal{F}}]\}$.

Proposition 2. $\left(\left(X_{1}^{*}, C_{1}^{*}\right), j\right)$ is a quasi-completion of $(X, C)$.

Proof. Clearly $\left(X_{1}^{*}, C_{1}^{*}\right)$ is a filter space and $j$ is a Cauchy embedding. To show that it is quasi-complete, let $\mathcal{A} \in C_{1}^{*}$. Then either $\mathcal{A} \geq j(\mathcal{F})$, for some $\mathcal{F}$ that is $q_{c}$-convergent or $\mathcal{A} \geq j(\mathcal{G}) \cap[\dot{\mathcal{G}}]$, for some $\mathcal{G}$ non- $q_{c}$-convergent. If $\mathcal{F} \stackrel{q_{c}}{\rightarrow} x$, then $j(\mathcal{F}) \stackrel{q_{c_{1}^{*}}}{\rightarrow} j(x)$. On the other hand, if $\mathcal{F}$ is non- $q_{c}$-convergent, then $j(\mathcal{F}) \cap[\dot{\mathcal{F}}] \in C_{1}^{*}$, which implies that $\mathcal{A} \stackrel{q_{c_{1} *}}{\rightarrow}[\mathcal{F}]$. Therefore, $\left(\left(X_{1}^{*}, C_{1}^{*}\right)\right)$ is quasicomplete. Next, let $[\mathcal{F}] \in X_{1} * \backslash j(X)$. This implies that $j(\mathcal{F}) \cap[\dot{\mathcal{F}}] \in C_{1}^{*}$, that is, $j(\mathcal{F}) \stackrel{q_{c_{1}^{*}}^{*}}{\rightarrow}[\mathcal{F}]$. Therefore, $[\mathcal{F}] \in c l_{q_{1}^{*}}(j(X))$. This proves that $\left(\left(X_{1}^{*}, C_{1}^{*}\right), j\right)$ is a quasi-completion of $(X, C)$, and this completes the proof.

This completion will be referred to as quasi-Wyler completion. Note that if $(X, C)$ is a $c$-filter space (respectively, Cauchy space), then $\left(\left(X_{1}^{*}, C_{1}^{*}\right), j\right)$ is a $c$-filter space (respectively, Cauchy space). If we identify each $x \in X$ with the equivalence class $[\dot{x}]$ of all filters which are $p_{c}$-convergent to $x$, then the quasiWyler completion coincides with $\left(\left(X^{*}, C^{*}\right), j\right)$ in [3]. We will refer to the latter completion as the $\mathrm{T}_{2}$ Wyler completion of $(X, C)$. Unlike $T_{2}$ completions of a filter space, the quasi-completion $\left(\left(X_{1}^{*}, C_{1}^{*}\right), j\right)$ is not a quasi- $T_{2}$ completion, in general, even if $(X, C)$ is $q-T_{2}$. The following proposition gives a condition which guarantees that a $q-T_{2}$ filter space has a quasi- $T_{2}$ completion.

Proposition 3. A $q-T_{2}$ filter space has a quasi- $T_{2}$ completion if and only if $(X, C)$ is a $c$-filter space.

Proof. Let $((Y, K), \phi)$ be a $q-T_{2}$ completion of $(X, C)$. Let $\mathcal{F} \in C$ and $\mathcal{F} \sim_{C}$ $\dot{x}$. From Proposition 1, it follows that $\phi(\mathcal{F}) \stackrel{q_{k}}{\rightarrow} \phi(\dot{x})$, that is, $\phi(\mathcal{F}) \cap \phi(\dot{x}) \in K$. Since $\phi$ is an embedding, $\mathcal{F} \cap \dot{x} \in C$, which shows that $(X, C)$ is a $c$-filter space.

Next, let $(X, C)$ be a $q-T_{2} c$-filter space. Then, as shown in Proposition 2, $\left(\left(X_{1}^{*}, C_{1}^{*}\right), j\right)$ is a quasi-completion of $(X, C)$. Let $\dot{y}_{1} \cap \dot{y}_{2} \in C_{1}^{*}$. If $y_{1}, y_{2} \in X$, 
then $y_{1}=y_{2}$, since $(X, C)$ is $q-T_{2}$. If at least one of $y_{1}$ or $y_{2}$ is in $X_{1}^{*} \backslash X$, then by the definition of $C_{1}^{*}, \dot{y}_{1} \cap \dot{y}_{1} \in C_{1}^{*}$ only when $y_{1}=y_{2}$. This completes the proof.

A quasi-completion $((Y, K), \phi)$ is said to be in standard form if $Y=X_{1}^{*}$ and $\phi=j$, satisfying the condition $j(\mathcal{F}) \stackrel{q_{c_{1} *}}{\rightarrow}[\mathcal{F}]$ for all non- $q_{c}$-convergent filters in $C$. A similar property was introduced by Reed [12] to establish that a $T_{2}$ Cauchy completion can be made equivalent to one in standard form. However, since this is not the case for all Cauchy spaces in general (see Example 3.2 [9]), the stable completions were introduced [9]. Since Cauchy spaces are special cases of filter spaces, Reed's result will also fail for non- $T_{2}$ filter spaces in general. This leads to the notion of quasi-stable completion for filter-spaces.

A quasi-completion $((Y, D), \phi)$ of a filter space $(X, C)$ is said to be quasistable if for each non- $q_{c}$-convergent filter $\mathcal{F} \in C, \phi(\mathcal{F}) \cap[\dot{\mathcal{F}}] \in D$. This property of a completion is stronger than the property of being stable introduced in [3], since quasi-stable implies that it is stable. However, there exist stable completion of some filter spaces which are not quasi-stable. Two quasi-stable completions of a filter space $(X, C)$ can be compared to each other in the obvious way: A quasi-stable completion $\left(\left(Y_{1}, K_{1}\right), \varphi_{1}\right)$ is said to be finer than another quasi-stable completion $\left(\left(Y_{2}, K_{2}\right), \varphi_{2}\right)$, if there is a continuous map $h:\left(Y_{1}, K_{1}\right) \rightarrow\left(Y_{2}, K_{2}\right)$ such that $h \circ \phi_{1}=\phi_{2}$, and they are equivalent if each is finer than the other. Note that the map $h$ is a unique homeomorphism, when the quasi-stable completions are equivalent.

Proposition 4. The quasi-Wyler completion is the finest quasi-stable completion in standard form.

Proof. Let $((Y, K), \phi)$ be a quasi-stable completion of the filter space $(X, C)$ and $h: Y \longrightarrow X_{1}^{*}$ be defined as

$$
h(y)= \begin{cases}{[\mathcal{F}]} & \text { if } y \in Y \backslash \phi(X) \text { and } \phi(\mathcal{F}) \stackrel{q_{k}}{\longrightarrow} y \\ y & \text { if } y=\phi(x) \text { for some } x \in X\end{cases}
$$

To show that $h$ is well-defined, let $y_{1}=y_{2} \in Y$. If $y_{1}=y_{2} \in \phi(X)$, then clearly $h\left(y_{1}\right)=h\left(y_{2}\right)$. If $y_{1}=y_{2} \in Y \backslash \phi(X)$, then $\phi\left(\mathcal{F}_{1}\right) \stackrel{q_{k}}{\longrightarrow} y_{1}$ and $\phi\left(\mathcal{F}_{2}\right) \stackrel{q_{k}}{\longrightarrow} y_{2}$, for which $\mathcal{G}_{1}=\phi^{-1}\left(\phi\left(\mathcal{F}_{1}\right) \cap \dot{y}_{1}\right)$ and $\mathcal{G}_{2}=\phi^{-1}\left(\phi\left(\mathcal{F}_{2}\right) \cap \dot{y}_{2}\right)$ are in $C$. This implies that $\mathcal{F}_{1} \vee \mathcal{G}_{1}, \mathcal{G}_{1} \vee \mathcal{G}_{2}$ and $\mathcal{G}_{2} \vee \mathcal{F}_{2}$ exist, which yields $\left[\mathcal{F}_{1}\right]=\left[\mathcal{F}_{2}\right]$. Therefore, $h\left(y_{1}\right)=h\left(y_{2}\right)$.

Next, let $h\left(y_{1}\right)=h\left(y_{2}\right)$. If $h\left(y_{1}\right)=x_{1}$ and $h\left(y_{2}\right)=x_{2}$ for $x_{1}, x_{2} \in X$, then $y_{1}=y_{2}$. On the other hand, if $h\left(y_{1}\right)=[\mathcal{F}]$ and $h\left(y_{2}\right)=[\mathcal{G}]$ for some $\mathcal{F}, \mathcal{G} \in C$, then $\mathcal{F} \sim_{c} \mathcal{G}$, which leads to $\phi(\mathcal{F}) \sim_{c} \phi(\mathcal{G})$. Therefore, $\phi(\mathcal{F}) \stackrel{q_{k}}{\longrightarrow} y_{1}$ 
and $\phi(\mathcal{G}) \stackrel{q_{k}}{\longrightarrow} y_{2}$, which imply $\phi(\mathcal{F}) \stackrel{q_{k}}{\longrightarrow} y_{1}, y_{2}$. But, since $((Y, K), \phi)$ is a quasistable completion of $(X, C)$, it follows that $y_{1}=y_{2}$. Hence, $h$ is bijective and $h \phi=j$.

Let $C^{\prime}=\{h(\mathcal{G}) \mid \mathcal{G} \in K\}$ be the quotient structure on $X_{1}^{*}$ with respect to $h$. Obviously, both $h$ and $h^{-1}$ are Cauchy maps, which makes the bijective maps $j$ and $j^{-1}$ Cauchy maps. It is also routine to show that $\left(X_{1}^{*}, C^{\prime}\right)$ is quasi-complete and $c l_{q_{c^{\prime}}} j(X)=Y$. Hence, $\left(\left(X_{1}^{*}, C^{\prime}\right), j\right)$ is a quasi-completion of $(X, C)$. This proves that $((Y, K), \phi) \simeq\left(\left(X_{1}^{*}, C^{\prime}\right), j\right)$. Also, for a non- $q_{c^{-c o n v e r g e n t ~ f i l t e r ~}}$ $\mathcal{F} \in C, \phi(\mathcal{F}) \stackrel{q_{k}}{\longrightarrow} y$ implies $j(\mathcal{F})=h \circ \phi(\mathcal{F}) \stackrel{q_{c^{\prime}}}{\longrightarrow} h(y)=[\mathcal{F}]$, which shows that $\left(\left(X_{1}^{*}, C^{\prime}\right), j\right)$ is in standard form. This completes the proof.

Note that the quasi-Wyler completion is the finest quasi-stable completion in standard form, but it is not the finest stable completion in FIL. In fact, there is no such finest one for a filter space [3], whenever $X^{*} \backslash j(X)$ is infinite.

\section{Extension Theorem}

Extension theorems for filter spaces [3], regular filter spaces [10], filter semigroups [11] and Cauchy spaces ( not necessarily $T_{2}$ ) [9] have led to some interesting reflective subcategories of the categories FIL and $C H Y$ with some special type of morphisms called $s$-maps. In case of $T_{2}$ filter spaces, an unique extension of a Cauchy map $f:(X, C) \longrightarrow(Y, D)$ to the corresponding completion space was possible only when the codomain was a $c$-filter space. Here, an extension theorem is established without this restriction on the codomain, which is a considerable departure from the previous results ([3], [4]).

A Cauchy map between two filter spaces $f:(X, C) \rightarrow(Y, D)$ is said to be a quasi-s-map, if it satisfies the following condition:

$\mathcal{F} \in C q_{c}$-converges to at most one point in $X$ implies that $f(\mathcal{F})$ is $D$-linked to at most one point in $Y$.

Note that a quasi-s map is an $s$-map [9]. There are several examples of quasi- $s$-maps. Any Cauchy map is a quasi-s-map, if the codomain of the map is a $q-T_{2}$ filter space. The identity map on a filter space and the embedding map $\varphi$ for a stable completion are also quasi-s-maps. In particular, the mapping $j$ in the quasi-Wyler completion is a quasi-s-map. Note that it follows from the definition of $s$-map that composition of two quasi- $s$-maps is a quasi- $s$-map. The class of all filter spaces with the quasi-s-maps as morphisms forms a category, which we call $F I L^{\prime}$. We observe that every Cauchy map is not necessarily a quasi-s-map. For example, any mapping from a nontrivial filter space or 
an incomplete filter space into an indiscrete filter space containing at least two points is a Cauchy map, but not a quasi-s-map. So $F I L^{\prime}$ is not a full subcategory of FIL.

The following proposition shows that the quasi-Wyler completion $\left(\left(X_{1}^{*}, C_{1}^{*}\right)\right.$, $j$ ) has a property similar to the universal property of the $T_{2}$ completions [3]. A significant departure from the previous result is that we don't need to restrict the codomain of the quasi-s-map to be a $c$-filter space [3].

Proposition 5. Let $(X, C)$ and $(Y, D)$ be two filter spaces with the quasi-Wyler completions $\left(\left(X_{1}^{*}, C_{1}^{*}\right), j_{X}\right)$ and $\left(\left(Y_{1}^{*}, D_{1}^{*}\right), j_{Y}\right)$, respectively. If $f:(X, C) \rightarrow(Y, D)$ is a quasi-s-map, then there is a unique extension $f^{*}$ : $\left(X_{1}^{*}, C_{1}^{*}\right) \rightarrow\left(Y_{1}^{*}, D_{1}^{*}\right)$ which is also a quasi-s-map and $f^{*} \circ j_{X}=j_{Y} \circ f$.

Proof. Define $f^{*}:\left(X_{1}^{*}, C^{*}\right) \rightarrow\left(Y_{1}^{*}, D^{*}\right)$ as follows

$$
\begin{gathered}
f^{*}(x)=f(x) \\
f^{*}([\mathcal{F}])=\left\{\begin{array}{cl}
{[f(\mathcal{F})]} & \text { if } f(\mathcal{F}) \text { not } D \text {-linked to } \dot{y} \text { for any } y \in Y, \\
y & \text { if } f(\mathcal{F}) \stackrel{q_{D}}{\longrightarrow} y \text { for some } y \in Y .
\end{array}\right.
\end{gathered}
$$

Note that $f(\mathcal{F})$ is not $D$-linked to $\dot{y}$ for any $y \in Y$ implies that $f(\mathcal{F})$ is $q_{D^{-}}$ non-convergent. The mapping $f^{*}$ is a well-defined map, because, if $[\mathcal{F}]=[\mathcal{G}]$, then $f(\mathcal{F}) \sim_{D} f(\mathcal{G})$. So either both $f(\mathcal{F})$ and $f(\mathcal{G})$ are not $D$-linked to any element in $Y$, or otherwise. In the first case, $f^{*}([\mathcal{F}])=f^{*}([\mathcal{G}])$. Otherwise, if $f(\mathcal{F}) \sim_{D} \dot{y_{1}}$ and $f(\mathcal{G}) \sim_{D} \dot{y_{2}}$, then $f(\mathcal{F}) \sim_{D} \dot{y}_{1}, \dot{y_{2}}$. This is a contradiction, since $\mathcal{F}$ is not $\mathrm{C}$-linked to $\dot{x}$ for any $x \in X$ implies $\mathcal{F}$ is $q_{c}$-non-convergent and $f$ is a quasi-s-map. So in either case $f^{*}([\mathcal{F}])=f^{*}([\mathcal{G}])$. Also, it can be easily verified that $f^{*} \circ j_{X}=j_{Y} \circ f$.

Next we show that $f^{*}$ is a quasi-s-map. Let $\mathcal{A} \in C^{*}$. If $\mathcal{A} \geq j_{X}(\mathcal{F})$, then $f^{*}(\mathcal{A}) \geq f^{*} \circ j_{X}(\mathcal{F})=j_{Y} \circ f(\mathcal{F}) \in D^{*}$. If $\mathcal{A} \geq j_{X}(\mathcal{F}) \cap[F]$, where $\mathcal{F}$ is not $C$-linked to any $x \in X$, then $f^{*}(\mathcal{A}) \geq\left(j_{Y} \circ f(\mathcal{F})\right) \cap f^{*}([\mathcal{F}])$. If $f(\mathcal{F})$ is $q_{D^{-}}$ non-convergent in $Y$, then $\left(j_{Y} \circ f(\mathcal{F})\right) \cap[f(\mathcal{F})] \in D^{*}$. If $f(\mathcal{F}) q_{D^{-c o n v e r g e s ~ t o ~}}$ $y \in Y$, then, $f(\mathcal{F}) \cap \dot{y} \in D$, so it follows that $\left(j_{Y} \circ f(\mathcal{F})\right) \cap \dot{y} \in D^{*}$. Therefore, $f^{*}$ is a Cauchy map. To show that it is a quasi-s-map, it suffices to show that

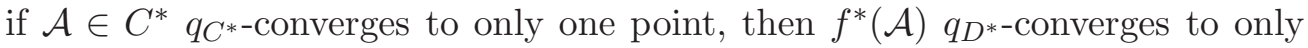
one point in $Y^{*}$. If $\mathcal{A} \geq j_{X}(\mathcal{F})$, then $j_{Y} \circ f(\mathcal{F})=f^{*} \circ j_{X}(\mathcal{F})$ is $D^{*}$-linked to

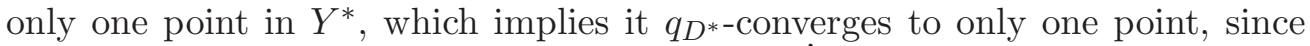
$j_{Y}$ and $f$ are quasi-s-maps. If $\mathcal{A} \geq j_{X}(\mathcal{F}) \cap[\dot{\mathcal{F}}]$, then $\mathcal{F}$ is not $C$-linked to any point in $X$, implies $\mathcal{F}$ is $q_{c}$-non-convergent. Hence, it follows from $f$ being a quasi-s-map that $f(\mathcal{F})$ is $D$-linked to at most one point in $Y$. Therefore, $\left.f^{*}\left(j_{X}(\mathcal{F}) \cap[\dot{\mathcal{F}}]\right)=\left(f^{*} \circ j_{X}(\mathcal{F})\right) \cap f^{*}(\dot{\mathcal{F}}]\right)=\left(j_{Y} \circ f(F)\right) \cap[f(\dot{\mathcal{F}})]$ or $\left(j_{Y} \circ f(\mathcal{F})\right) \cap \dot{y}$ according as $f(\mathcal{F})$ is not $D$-linked to any point (hence $q_{D}$ non-convergent) or 
$f(\mathcal{F}) q_{D^{-}}$-converges to $y \in Y$. But in either case $f^{*}(\mathcal{A})$ converges to only one point in $Y^{*}$.

Finally, we show that $f^{*}$ is an unique extension. Let $\bar{f}:\left(X^{*}, C^{*}\right) \rightarrow$ $\left(Y^{*}, D^{*}\right)$ be another quasi-s-map such that $\bar{f} \circ j_{X}=j_{Y} \circ f$. It is obvious that $\bar{f} \circ j_{X}(x)=f^{*} \circ j_{X}(x)$ for all $x \in X$. So, let $[\mathcal{F}] \in X^{*} \backslash j_{X}(X)$. Since $\mathcal{F} \in C$ is not $C$-linked to any point in $X, j_{X}(\mathcal{F}) \cap[\dot{\mathcal{F}}]$. Since $f^{*}, \bar{f}$ are also Cauchy maps,

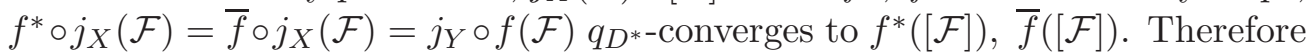
$j_{Y} \circ f(\mathcal{F})$ is $D^{*}$-linked to both $f^{*}([\mathcal{F}])$ and $\bar{f}([\mathcal{F}])$. However, $\mathcal{F}$ is not $C$-linked, which implies it is also $q_{c}$-non-convergent, and $f, j_{Y}$ are quasi-s-maps imply that $j_{Y} \circ f(\mathcal{F})$ can be $D^{*}$-linked to at most one point in $Y^{*}$. Hence $f^{*}=\bar{f}$. This completes the proof.

The unique mapping $f^{*}$ in Proposition 5 is called the quasi-s-extension of $f$.

Remark (I) If $f:(X, C) \rightarrow(Y, K)$ is a quasi-s-map, where $(Y, K)$ is a quasi-complete filter space, then there exists a unique quasi-s-extension $f^{*}$ : $\left(X^{*}, C^{*}\right) \rightarrow(Y, K)$ such that $f^{*} \circ J_{X}=f$.

(II) If $(X, C)$ is a $q-T_{2}$ filter space, then its $T_{2}$ quasi-Wyler completion also has the extension property. Recall that if the codomain of an $s$-map is a $q-T_{2}$ space, then the $s$-map is simply a Cauchy map. If $f:(X, C) \rightarrow(Y, K)$ is a Cauchy map, where $(Y, K)$ is a complete $T_{2} c$-filter space [3] (or a complete $T_{3}$ filter space [10]), then there exists a unique Cauchy extension $f^{*}:\left(X^{*}, C^{*}\right) \rightarrow$ $(Y, K)$ such that $f^{*} \circ J_{X}=f$.

Note that a composition of quasi-s-maps is a quasi-s-map and the identity map is a quasi-s-map. So the class of all filter spaces with quasi-s-maps as morphisms form a subcategory of FIL. We denote this category by $F I L^{\prime}$. Since it comprises quasi-s-maps as morphisms, it is not a full subcategory of $F I L$. Let $F I L^{\prime *}$ be the subcategory of $F I L^{\prime}$ consisting of the quasi-complete objects of $F I L^{\prime}$. On the category $F I L^{\prime}$, we can define a functor $W_{q}: F I L^{\prime} \rightarrow F I L^{\prime *}$ by $W_{q}(X, C)=\left(X_{1}^{*}, C_{1}^{*}\right)$ for all objects, and $W_{q}(f)=f^{*}$ for all morphisms in $F I L^{\prime}$. Using the property of $s$-maps, it is a routine matter to show that $W_{q}$ is a covariant functor on $F I L^{\prime}$. The functor $W_{q}$ is called the quasi-Wyler completion functor.

\section{References}

[1] H.L. Bently, H. Herrlich, E. Lowen-Colebunders, Convergence, J. Pure Appl. Algebra, 68 (1990), 27-45; doi: 10.1016/0022-4049(90)90130-A. 
[2] M. Katetov, On continuity structures and spaces of mappings, Comment. Math. Carolinae, 6 (1965), 257-278; https://eudml.org/doc/16128.

[3] D.C. Kent, N. Rath, Filter spaces, Applied Categorical Structures, 1 (1993), 297-309; doi: 10.1007/BF00873992.

[4] D.C. Kent, N. Rath, On completions of filter spaces 767 , Annals of the New York Academy of Sciences (1995), 97-107; doi: 10.1111/j.17496632.1995.tb55898.x.

[5] G. Minkler, J. Minkler, G. Richardson, Extensions for filter spaces, Acta. Math. Hungar., 82, No. 4 (1999), 301-310; doi: 10.1023/A:1006688224938.

[6] G. Preuss, Semiuniform convergence spaces and filter spaces, (Beyond Toplogy, Contemporary mathematics Series- 486, AMS Publn., 2009, Eds: F. Maynard and E. Pearl) 333-374.

[7] G. Preuss, Improvement of Cauchy spaces, QESA in General Topology, 9 (1991), 159-166.

[8] G. Preuss, Theory of Topological Structures, D. Reidel Publ. Co., Dordrecht (1988).

[9] N. Rath, Completion of a Cauchy space without the $T_{2}$ restriction on the space, Int. J. Math. Math. Sci., 24, No. 3 (2000), 163-172, doi: 10.1155/S0161171200003331.

[10] N. Rath, Regular filter spaces, Topics in Applied Theoretical Mathematics and Computer Science, WSES Press (2001) 249-254; http://www.wseas.us/e-library/conferences/cairns2001/papers/610.pdf.

[11] N. Rath, Completions of filter semigroups, Acta. Math. Hungar., 107, No-s: 1-2 (2005), 45-54; http://link.springer.com/article/10.1007/s10474005-0176-0.

[12] E.E. Reed, Completions of uniform convergence spaces, Math. Ann., 194 (1971), 83-108; doi: 10.1007/BF01362537. 